\title{
Circadian rhythm of renal blood flow in caged sheep. Influence of eating and rumination*
}

\author{
N. Denis ${ }^{2}$, I. Tebot ${ }^{1}$, J.-M. Bonnet ${ }^{3}$ and A. Cirio ${ }^{1,4}$ \\ ${ }^{1}$ Departamento de Fisiología, \\ ${ }^{2}$ Departamento de Rumiantes y Suinos, Facultad de Veterinaria \\ Montevideo 11600, Uruguay \\ ${ }^{3} U M R$ Laboratoire de Physiologie, Ecole Nationale Vétérinaire de Lyon \\ BP 83, F-69280 Marcy L'Etoile, France
}

\begin{abstract}
A renal blood flow (RBF) circadian rhythm and its relationship with eating and rumination was described in six once daily fed (09.00-12.00 h) caged sheep, using ultrasonic flowmeters bilaterally implanted around renal arteries. Following a rapid raise during the first $20 \mathrm{~min}$ after the start of feeding, the RBF progressively increased until $15.00 \mathrm{~h}$ ( $14 \%$ maximum increase, $\mathrm{P}<0.05$ from 12.10 to $20.50 \mathrm{~h}$ compared with pre-feeding values $(519 \mathrm{ml} / \mathrm{min}))$. After $15.00 \mathrm{~h}$, the RBF regularly decreased reaching its minimum values before the next feeding time. An effect of rumination on RBF circadian rhythm was not observed.
\end{abstract}

KEY WORDS: renal blood flow, circadian rhythm, rumination, eating, sheep

\section{INTRODUCTION}

A circadian rhythm in renal blood flow (RBF) and a strong association of its profile with feeding behaviour have been reported in rats (Gehrig et al., 1986; Labrecque et al., 1988) and rabbits (Barrett et al., 2001). Ruminants can adapt their RBF to meal composition (i.e. decrease in response to protein restriction reducing the elimination of urea, a useful precursor of microbial protein synthesis in the forestomachs; Cirio and Boivin, 1990). For a better understanding of these adaptive mechanisms, it is helpful to establish the relationship between feeding patterns and RBF. The aim of this work was to determine the existence of a

\footnotetext{
* Supported by the Direction Générale de l'Enseignement et de la Recherche (France) and by the Alfa Program, European Commission and Universidad de la República, Uruguay, Grant 6.0217.9

${ }^{4}$ Corresponding author: e-mail: cirio@montevideo.com.uy
} 
circadian rhythm of RBF in adult caged sheep and its association with eating and rumination times.

\section{MATERIAL AND METHODS}

RBF was continuously recorded during 6 days in 6 adult caged Texel ewes (50-60 kg BW), fed between 09.00-12.00 h, by means of ultrasonic flowmetering probes ( $4 \mathrm{~mm}$, R-series, Transonic Systems, USA) bilaterally implanted around renal arteries. To monitor feeding behaviour, chewing was recorded by the submandibular balloon technique. Data were treated by a biological signals processing system (Acqknowledge III, Biopac Systems, USA). Mean values of RBF were calculated every ten minutes. At the end of the experiments the implanted probes were validated for zero blood flow and calibrated in order to verify the identity between the real and the measured flows. Care was taken to perform the experiments in accordance with international ethical guidelines.

\section{RESULTS}

A circadian rhythm of RBF in caged sheep was observed (Figure 1). Following a rapid raise during the first 20 min consecutive to the onset of feeding, the RBF progressively increased until $15.00 \mathrm{~h}$ (about 6 hours after the start of eating, $14 \%$ maximum increase). The difference with pre-feeding values was statistically significant from 12.10 to $20.50 \mathrm{~h}$. After $15.00 \mathrm{~h}$, the $\mathrm{RBF}$ regularly decreased reaching its minimum values before the next feeding time. An effect of rumination on RBF circadian rhythm was not evident. The successive rumination periods or isolate events, occurring after the end of the

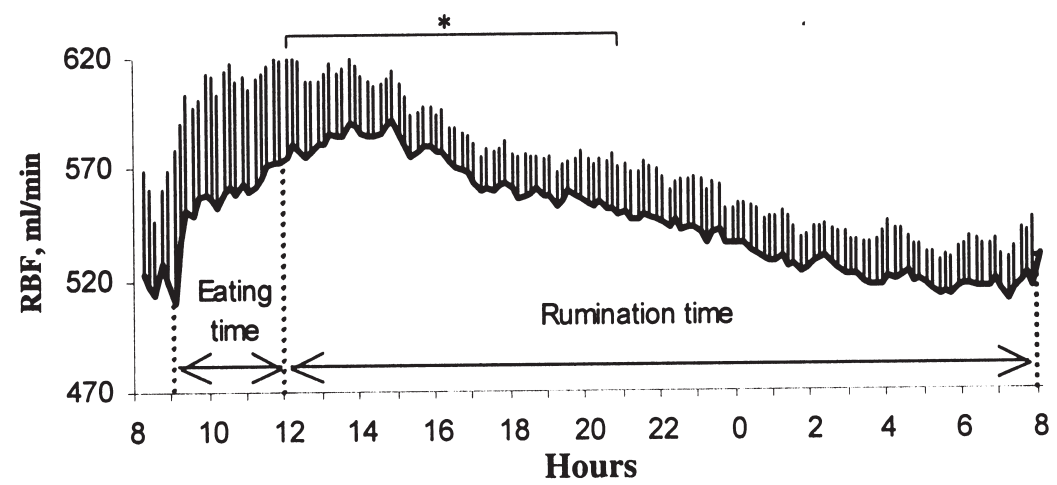

Figure 1. Circadian changes in renal blood flow (RBF) and feeding behaviour in caged sheep. Only infrequent rumination events were observed between 08.00 and $12.00 \mathrm{~h}$. Values are means \pm SEM over 10 min for both kidneys. * $\mathrm{P}<0.05$ compared to pre-feeding $(08.00-09.00 \mathrm{~h})$ mean value, $\mathrm{n}=6$ sheep $(\times 6$ days each), Student $t$ test (Intercooled Stata V.80) 


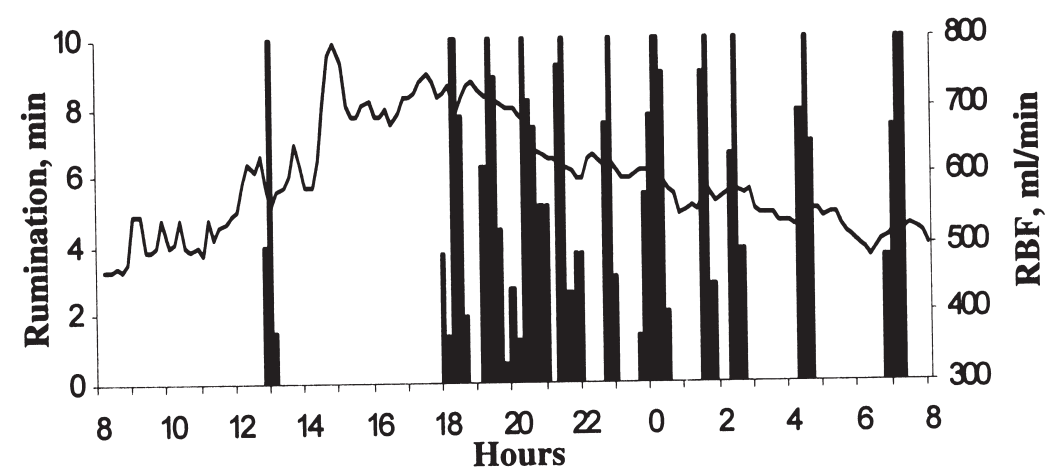

Figure 2. Typical 24-h recording of renal blood flow changes (RBF, continuous line) and rumination behaviour (vertical bars). Each RBF data is the mean value over $10 \mathrm{~min}$ for both kidneys. Bars represent the time spent on rumination chewing every $10 \mathrm{~min}$

meal (rumination time, Figure 1), did not modify the regular decreasing profile of the RBF plotting. A detailed typical daily pattern of rumination, not affecting the RBF, is showed on Figure 2.

\section{DISCUSSION}

The results disclosed a 24-h rhythm in RBF of once daily fed caged sheep. This rhythm seems to be related to feeding time since a rapid increase in RBF followed the start of eating $(09.00 \mathrm{~h})$ in all the animals and, after its maximum value (acrophase, $15.00 \mathrm{~h}$ ), the flow regularly decreased until the next feeding time. On the contrary, we were unable to show any change of RBF associated with rumination. This fact could be interpreted, in an evolutionary sense, as the link of RBF rhythm to more primitive feeding patterns (food ingestion, pre-ruminant stage) rather than to more recent ones (rumination, adult stage). In a previous work (Silva et al., 1993), a reduction in glomerular filtration rate during 20-min eating in caged sheep was observed, suggesting changes in RBF opposite to those reported here. As the authors did not measure the RBF, it could be argued that the reduction in filtration rate was independent of RBF values. Nevertheless, the reason for the difference between the two studies remains unclear.

The dependence of the RBF circadian rhythm on feeding behaviour is strongly suggested in non-ruminant species. In rats, the acrophase of the 24-h rhythm of RBF has been found during the active periods of animals, coincident with their ingestive behaviour (Labrecque et al., 1988). Gehrig et al. (1986) reported that alternate-day feeding causes significant oscillations in renal haemodynamics, feeding leading to a 19\% increment in RBF compared to fasting days. In rabbits, Barret et al. (2001) observed a RBF 24-h rhythm dependent on feeding time: the circadian profile (very similar to ours) increased at the onset of eating $(09.00 \mathrm{~h})$, reached the maximum values 
near midday and progressive decreased over the next $12 \mathrm{~h}$. The shift of the feeding time to $15.00 \mathrm{~h}$, with the corresponding shift in the RBF circadian profile, confirmed the origin of the rhythm. The only work we found reporting a circadian rhythm of RBF in ruminants was performed in near-term ovine foetus (Braaksma et al., 2000). The circadian profile (acrophase at $01.45 \mathrm{~h}$ ) was independent of feeding time since the mother was fed ad libitum. The authors concluded that the origin of the foetal RBF rhythm is actually not known.

More studies are necessary to assess the role of feeding time and/or the internal biological clock on the determination of this rhythm in sheep. Glucagon should not be excluded as a possible mediator considering a. that the circadian rhythm of its pancreatic release depends on feeding behaviour in monogastric animals (Ruiter et al., 2003), b. that amino acids increased the glucagonaemia in sheep (Kuhara et al., 1991) and c. that the intravenous infusion of physiological doses of glucagon induced a highly sensitive dose-dependent increase in RBF in sheep (Denis et al., 2003). In anyway, it must be kept in mind that present results cannot be extrapolated to grazing sheep because of their natural continuous feeding behaviour.

\section{CONCLUSIONS}

A circadian rhythm of RBF was observed in once daily fed caged sheep. Its profile seemed to be associated with eating time but not with rumination periods.

\section{REFERENCES}

Barrett C.J., Navakatikyan M.A., Malpas S.C., 2001. Long-term control of renal blood flow: what is the role of the renal nerves? Amer. J. Physiol. - Regul. Integr. C 280, R1534-1545

Braaksma M.A., Poortinga F.M.I., Aarnoudse J.G., 2000. Daily rhythm in renal blood flow and urine production rate in the near-term sheep fetus. Pediat. Res. 47, 773-777

Cirio A., Boivin R., 1990. Urea recycling from the renal pelvis in sheep: a study with $\left[{ }^{14} \mathrm{C}\right]$ urea. Amer. J. Physiol. 258, F1196-1202

Denis N., Tebot I., Bonnet J.M., Cirio A., Boivin R., 2003. Effects of intravenous perfusion of glucagon on renal blood flow in conscious sheep. Exp. Physiol. 88, 575-580

Gehrig J.J. Jr., Jamison R.L., Baylis C., Troy J.L., Brenner B.M., Jamison R.L., 1986. Effect of intermittent feeding on renal hemodynamics in conscious rats. Amer. J. Physiol.- Renal Physiol. 250, F566-572

Kuhara T., Ikeda S., Ohneda A., Sasaki Y., 1991. Effects of intravenous infusion of 17 amino acids on the secretion of GH, glucagon and insulin in sheep. Amer. J. Physiol. 260, E21-26

Labrecque G., Belanger P.M., Dore F., Lalande M., 1988. 24-hour variations in the distribution of labeled microspheres to the intestine, liver and kidneys. Annu. Rev. Chronopharmacol. 5,445-448

Ruiter M., La Leur S.E., van Heijningen C., van der Vliet J., Kalsbeek A., Buijs R.M., 2003. The daily rhythm in plasma glucagon concentrations in the rat is modulated by the biological clock and by feeding behavior. Diabetes 52, 1709-1715

Silva R., Tebot I., Rodríguez M., Cirio A., 1993. Existence d'un réflexe antidiurétique pendant la prise alimentaire chez le mouton. Vet. Res. 24, 89-92 